\title{
Entre la libertad de elección y las preferencias adaptativas. Reflexiones desde la teoría feminista
}

\author{
Between Freedom of Choice and Adaptative Preferences. \\ Reflections from Feminist Theory
}

\section{Resumen}

En el presente trabajo propongo abordar el debate en torno a las preferencias adaptativas. Esta cuestión, analizada ampliamente desde diversas perspectivas feministas, es fundamental para comprender las discusiones y propuestas teóricas en el marco de los debates sobre la libertad de las mujeres. En este sentido, intentaré reflexionar acerca de la posibilidad de cuestionar algunas de las elecciones de aquellas personas que se encuentran en situaciones subalternizadas o de subordinación. Sin embargo, al mismo tiempo que propongo abordar el modo en que las preferencias son moldeadas por las opciones que tenemos a disposición (Hampton, 1993; Okin, 1996, 2003; Nussbaum, 2002), pretendo no perder de vista los peligros que enfrentamos al poner en cuestión las expectativas, deseos y decisiones de las mujeres y otres sujetes subalternizades (Jaggar, 2006; Phillips, 2009).

Palabras clave: feminismos, libertad, preferencias, adaptación, elección.

\begin{abstract}
In this paper I propose to address the debate around adaptive preferences. This issue, analyzed extensively from various feminist perspectives, is essential to understand the theoretical discussions and proposals within the framework of women's freedom debates. In this sense, I will try to reflect on the possibility of questioning some of the choices of those people who are in subalternized or subordinate situations. However, while I propose to address the way in which preferences are shaped by the options we have available (Hampton, 1993; Okin, 1996, 2003; Nussbaum, 2002), I intend not to lose sight of the dangers we face when putting in question the expectations, desires and decisions of women and other subalternized subjects (Jaggar, 2006; Phillips, 2009).
\end{abstract}

Keywords: Feminisms, freedom, preferences, adaptation, choice.

\section{SUMARIO}

1.- ¿Decidir libremente? 2.- Autoras de nosotras mismas. 3.- Adaptarnos al contexto. 4.Preferir y elegir no son lo mismo. 5.- Para concluir, tensiones. Referencias bibliográficas.

\section{1. ¿Decidir libremente?}

La cuestión que propongo abordar en primer lugar es hasta qué punto podemos hablar de elecciones o decisiones libres de las mujeres en el marco de estructuras

1 Consejo Nacional de Investigaciones Científicas y Técnicas (CONICET), Instituto de Investigaciones en Estudios de Género de la Universidad de Buenos Aires (Argentina), anabella.ditullio@gmail.com 
patriarcales, de una socialización sexista, de opresión y de desvalorización de todo lo emparentado con lo «femenino». Aquí deberíamos reflexionar por un lado la cuestión de las preferencias adaptativas, o dicho en otras palabras, el modo en el que el contexto moldea los deseos y elecciones de las personas; y por el otro, íntimamente relacionado con el punto anterior, el lugar que ocupan en estos desarrollos teóricos las experiencias y las voces de las mujeres reales, en su diversidad y heterogeneidad.

Las preferencias en las que basamos nuestras decisiones se encuentran adaptadas a contextos sociales, culturales, económicos y políticos. El sistema de género en el que nos socializamos, opera moldeando intereses y expectativas en función de los estereotipos de género que prevalecen en las sociedades contemporáneas: actitudes, valores, habilidades y preferencias son distribuidos en base al sexo de cada persona desde su nacimiento. En este sentido, M. Jacqui Alexander y Chandra Talpade Mohanty afirman:

Por ejemplo, la reciente difusión dela cultura del consumo, siempreeurocéntrica, en consonancia con una mayor consolidación del capital multinacional, pone en primer plano la necesidad de teorizar las formas en las que la desigualdad estructura los valores, los deseos y las necesidades de los diferentes grupos y clases de mujeres. Cualquier comprensión de las experiencias de las mujeres que se base en una concepción estrecha del género sería simplemente incapaz de enfocar los efectos homogeneizadores y jerarquizadores de los procesos económicos y culturales, que son el resultado de esta cultura de consumo (Alexander y Mohanty, 2004: 142).

Las preguntas en torno a la libertad o autonomía al momento de hacer elecciones y tomar decisiones, cobran un sentido especial en el marco de un feminismo como el liberal, pues las autoras que se sitúan en esta corriente se muestran especialmente comprometidas con los valores de la libre y autónoma elección de cada individuo. El liberalismo sostiene un total compromiso con las decisiones y elecciones de las personas, pues reflejan el modo en que cada una interpreta el bien y escoge su propio plan de vida. Puede resultar, entonces, particularmente interesante -y disonante- cuando estas teóricas sostienen la idea de que las preferencias de las personas que se encuentran en situaciones de subordinación se adaptan en algún sentido a esa condición subordinada. Es por ello que propongo que nos centremos en primer lugar en algunos postulados de autoras que se reconocen dentro del feminismo liberal, para analizar qué herramientas nos ofrecen para abordar la cuestión que nos ocupa y qué problemáticas nos plantean. Me centraré en las reflexiones de feministas liberales como Jane Hampton, Susan Moller Okin, Martha Nussbaum y Ann Cudd, para indagar cuál es su abordaje en torno a la tensión entre la idea de preferencias adaptativas y de libre elección. Hacia el final, intentaré mostrar el modo en que otras miradas feministas críticas del liberalismo -Catharine MacKinnon y Nancy Hirschmann-, nos permiten un abordaje diferente sobre la temática, y nos brindan mayores herramientas para abordar esta aporía. 


\section{Autoras de nosotras mismas}

La filósofa Jane Hampton afirma que para que una preferencia sea considerada de propia autoría (self-authored) debe estar definida subjetivamente. La idea de autoría en Hampton responde a las decisiones y elecciones propias de una persona que moldean y dan forma a sus preferencias y valores, más allá de la biología, la genética, o el ambiente. Pero esta condición, necesaria aunque no suficiente, ha de ir acompañada por un contenido específico de esa preferencia: no todo lo que una persona decide, desea o prefiere cuenta como una preferencia atribuible a ese «yo». Hampton argumenta que «es condición necesaria para que una preferencia sea auto-creada, que su contenido no entre en conflicto con aquello que se requiere para satisfacer las necesidades objetivas de esa persona en tanto ser humano» (Hampton, 1993: 57)2. Las preferencias autolesivas o autodestructivas quedan, entonces, fuera de la clasificación de Hampton.

Del mismo modo, las preferencias fundadas en aquello que una persona cree que es lo que se espera socialmente de ella, o que ese es el rol o lugar que debe ocupar en la sociedad y lo acepta pasivamente, no pueden considerarse auténticas; es decir, no son un proyecto auténtico de nuestra autoría. Esto no quita, para Hampton, responsabilidad sobre la decisión, sino que señala que esta no ha sido realmente una genuina «autoexpresión». Es esa posibilidad de crear(se), expresar(se) y definirse una misma la que hace que el desarrollo y «florecimiento» de los seres humanos/as sea tan diverso como diferentes personas hay en el mundo ${ }^{3}$. Solo mediante las preferencias que Hampton entiende como auténticas podemos considerar a las personas como autoras de sí mismas. Analicemos, para ilustrar esta idea hamptiana, el ejemplo que la autora desarrolla en «Selflessness and Loss of Self»:

El año pasado, una amiga hablaba conmigo sobre una conocida cuyos/as dos hijos/as estaban en la misma escuela infantil que nuestros/as hijos/as. Esta mujer, a quien llamaré Terry, había estado embarazada de gemelos, pero uno de los gemelos había muerto durante el cuarto mes de gestación, y el otro gemelo acababa de nacer prematuramente a los seis meses con un montón de problemas médicos. Estábamos conversando acerca de lo estresante que había sido la vida de esta mujer mientras estaba embarazada: era ama de casa, y sus dos hijos, de tres y cinco años de edad, eran vivaces, desafiantes, a menudo rebeldes -niños realmente difíciles de criar. Su marido trabajaba largas horas en un bufete de abogados, por lo que la gran mayoría del cuidado de los hijos y las tareas del hogar caían sobre sus hombros. «Se podía ver que estaba agotada hacia el final del primer trimestre», sostuve, «sus ojos estaban cansados y sus mejillas hundidas, se veía casi como un cadáver». Mi amiga estuvo de acuerdo. Seguí la conversación culpando de su agotamiento al hecho de que tuvo que hacer demasiado durante un embarazo que cualquiera habría encontrado difícil. «No entiendo a su marido», afirmé. «Seguramente podía ver lo mal que estaba. Si se preocupaba por sus futuros hijos,

2 Todas las traducciones son propias.

3 Hampton es consciente de que su concepción asume un carácter idealista sobre el florecimiento humano y sobre lo que significa ser un ser humano distintivo y particular (Hampton, 2007: 59). 
¿por qué no hizo algo para ayudarla para que el embarazo tuviera la oportunidad de ir mejor? Y si la amaba, ¿por qué no redujo sus horas para poder ayudarla en la casa? Seguramente, con solo mirarla, podía ver que se encontraba en problemas». Mi amiga no dijo nada en ese momento, pero después de una semana me llamó y me dijo que mi crítica al marido de esta mujer la había molestado durante toda la semana. «Te equivocas sobre el marido de Terry no preocupándose lo suficiente por ella. Tienen un buen matrimonio», insistió, y luego continuó: «Tú no eres como nosotras. Nosotras aceptamos el hecho de que tenemos que realizar la mayor parte del cuidado de los/as niños/as y de las tareas domésticas. El marido de Terry no estaba haciendo nada malo esperando que ella asumiera la responsabilidad de ese tipo de cosas» (Hampton, 1993: 39-40)4.

Más allá de las implicancias en cuanto a la distribución sexual del trabajo que este ejemplo muestra, lo que interesa resaltar aquí es que las preferencias de Terry no pueden ser calificadas como auténticas, pues sus elecciones son para Hampton, autodestructivas. Y aunque Terry asegurara su pasión por el cuidado de sus hijos, las tareas domésticas o la labor de beneficencia, deberíamos desde esta perspectiva, sospechar de un entusiasmo que se deposita justamente en aquellas tareas que se supone que las mujeres de su grupo social deben realizar. ¿No podríamos pensar entonces que toda elección que se enmarque en ciertos roles tradicionales es inauténtica? Cuando elegimos una carrera determinada, o establecer un vínculo sexo afectivo específico, o maternar/paternar, todes estamos en algún punto acogiéndonos a roles, en cierto modo, esperables. Lo que haría en el caso de Terry que no pueda ser considerada una autora de sí misma a los ojos de Hampton, pareciera ser el hecho de que el rol que acepta desarrollar convierte a su esposo e hijos en sus únicos fines, y a ella misma en un instrumento para el cuidado y sostenimiento de esa familia. Terry no se encontraría en condiciones de cubrir sus necesidades como ser humano ni de encontrar espacios de autoexpresión o autodefinición -como requisitos de la autoría de sí misma- fuera de su rol de madre y esposa.

Ahora bien, más allá de estas salvedades, lo cierto es que resulta difícil encontrar en el planteo de Hampton preferencias que pudieran ser consideradas como autónomas desde sus exigentes estándares. Sabemos que la mayoría de las mujeres, a pesar de trabajar largas horas fuera de su hogar, siguen siendo las cuidadoras principales de los miembros de su familia. Hampton analiza esta situación en términos de fenómeno social: estas decisiones se toman para evitar la desaprobación del entorno familiar, del grupo de amigos o de la iglesia a la que se pertenezca. En este sentido, «incluso si Terry hubiera redefinido su papel en la casa para que sus necesidades objetivas pudieran satisfacerse, seguiremos rechazando la idea de que la elección era auténtica si percibimos que es una elección que hizo con el fin de evitar tal desaprobación social» (Hampton, 1993: 60). No se trata entonces de su conducta autolesiva sino de unas decisiones tomadas en base a las expectativas de les otres y no de los propios deseos o necesidades.

4 Este ejemplo también es un disparador para Hampton en la discusión en torno a la idea de cuidados, y sus reflexiones en torno al sacrificio y al altruismo. 
Todas las personas sufrimos presiones a partir de expectativas sociales sobre nuestras conductas, deseos y preferencias. Para ser consideradas como autoras de nosotras mismas, deberíamos decidir en base a atributos, intereses y proyectos consistentes con el desarrollo de nuestras potencialidades como seres humanos, pero sobre todo, deseados, queridos y elegidos por nosotras mismas. Pero en este marco, cabe aclarar, la responsabilidad es para Hampton tanto individual como social: es necesaria una sociedad preparada para respetar opciones que se alejan de los estándares, pero que debe además garantizarles a las personas lo necesario para que sean capaces de comprometerse en la tarea de ser autoras de sí mismas. No obstante, el modo en el cual evaluaríamos la autenticidad de las preferencias sigue siendo por demás confuso y problemático. ¿Quién decide sobre la autoría o autenticidad de las preferencias de las personas? ¿Desde qué lugar -libre de esas presiones y expectativas sociales- puede juzgar alguien las preferencias de otres?

\section{Adaptarnos al contexto}

Cargadas con las preguntas y complejidades que en Hampton no hemos podido desentrañar, propongo que intentemos despejar algunos interrogantes a través del pensamiento de la filósofa Martha Nussbaum. Esta autora contempla también el hecho de que las preferencias de las personas están sometidas a distintos grados de manipulación -social, estatal y/o cultural-, y afirma que en muchas ocasiones los individuos pertenecientes a grupos subalternizados internalizan su situación de inferioridad y toman decisiones que no hacen sino reforzar y prolongar esa posición desfavorable. Si las preferencias están construidas, en parte, por las condiciones sociales y económicas, por las tradiciones, las relaciones de privilegio y de subordinación, «una perspectiva basada en las preferencias generalmente reforzará las desigualdades: especialmente aquellas desigualdades que están tan arraigadas que se han integrado a los propios deseos de las personas» (Nussbaum, 2009: 105).

¿Pues cómo evitar ese agravamiento de las desigualdades? Una de las formulaciones más reconocidas de la autora consiste en una teoría con pretensión de disputarle espacio a los métodos hegemónicos de medición del desarrollo humano. Nussbaum retoma dos conceptos centrales de la teoría de Rawls para construir su propuesta del «enfoque de las capacidades»: la noción de liberalismo político y de consenso traslapado. Con este objetivo, elabora una lista de capacidades humanas centrales, las cuales, en el contexto de un liberalismo político «rawlsiano» ${ }^{5}$, aparecen como metas netamente políticas -libres de fundamentaciones metafísicas-, propiciando así la posibilidad de establecer consensos entre personas que tengan o puedan tener distintas nociones del bien.

El enfoque de las capacidades propuesto por la autora es presentado «como base filosófica para una teoría de los derechos básicos de los seres humanos que deben ser respetados y aplicados por los gobiernos de todos los países, como requi- 
sito mínimo del respeto por la dignidad humana» (Nussbaum, 2007: 83) ${ }^{6}$. El objeto de la propuesta es centrar nuestra atención en las capacidades humanas, definidas por Nussbaum como «aquello que las personas son efectivamente capaces de hacer y ser, según una idea intuitiva de lo que es una vida acorde con la dignidad del ser humano» (Nussbaum, 2007: 83), para plantear, a partir de allí, la idea de un mínimo social básico de justicia. Puede resultar problemático, sin embargo, que luego de haber reconocido la dificultad que tienen los seres humanos para ponerse de acuerdo en torno a una idea comprehensiva del bien, Nussbaum acepte el «intuitivo» carácter universal de la definición de dignidad del ser humano (Di Tullio, 2007).

Siguiendo el enfoque propuesto por Nussbaum, la pregunta que debería orientarnos no es cuán satisfecha está una persona con aquello que hace, ni qué cantidad de recursos es capaz de producir esa persona, sino más bien, ¿qué es esa persona realmente capaz de hacer y de ser? Se trata principalmente de plantear objetivos políticos que deberían considerarse fundamentales para el desarrollo de la vida humana.

El enfoque filosófico de Nussbaum toma a cada individuo como su unidad básica de análisis, y entiende a cada uno como un fin en sí mismo. Desde esta perspectiva, serían solo los individuos (y no los grupos) quienes podrían considerarse como portadores de derechos. La teórica feminista Susan Moller Okin critica este aspecto del enfoque de Nussbaum, sosteniendo que los grupos también poseen capacidades, y que incluso muchas de las capacidades que conforman la lista de Nussbaum, dependen de hecho de la vida en grupo en diversas formas para su realización. Pero a la vez que pareciera que Nussbaum no toma en cuenta lo suficiente estas relaciones con les otres en sus diversos ámbitos de pertenencia, también es acusada de no darle relevancia a la voz propia e individual de las mujeres de países en desarrollo, a pesar de reclamar la urgencia de establecer un diálogo con ellas: «Parece extraño, por lo tanto, que en un libro de trescientas páginas, cada una de las mujeres de la India a quienes entrevistó -Vasanti y Jayamma- habla por sí misma, siendo directamente citada sólo una vez. De Jayamma, dice «no parece interesada en hablar», lo que hace que una se pregunte acerca de su elección como sujeta a ser entrevistada» (Okin, 2003: 295) ${ }^{7}$.

6 Este enfoque surge como alternativa a posiciones económico-utilitaristas en el contexto de los debates internacionales sobre desarrollo humano, y ha sido desarrollado por Martha Nussbaum en el ámbito de la filosofía y por Amartya Sen en el ámbito de la economía y las ciencias sociales (aunque en los últimos años también ha incursionado en la filosofía). Sen promueve la noción de capacidad como herramienta para la medición y comparación de la calidad de vida de las personas a nivel mundial, rivalizando con otras formas de medición como el PIB per cápita o la utilidad. El economista sostiene que de este modo pueden plantearse más adecuadamente las preguntas acerca de la igualdad y la desigualdad social. Cfr. Sen, 1998, 2011.

7 Okin se refiere aquí a Las mujeres y el desarrollo humano (2002). Cabe destacar que en el más reciente libro de Nussbaum sobre el tema de las capacidades (Crear capacidades, 2012), la autora vuelve a narrar la historia de Vasanti, pero llega nuevamente al mismo punto donde lo había dejado en el primer libro: con ayuda de la ONG SEWA (Self Employed Women's Association) Vasanti había obtenido un crédito que le permitió saldar la deuda con sus hermanos, había devuelto ese crédito en pocos años, reunía los requisitos necesarios para matricularse en los proyectos educativos de SEWA para aprender a leer y a escribir, y trabajaba contra la violencia hacia las mujeres en su comunidad. Dado que pasaron 11 años entre un libro y otro, a quienes seguimos el trabajo de Nussbaum nos hubiera resultado interesante conocer qué sucedió con Vasanti en esos años, y que su historia apareciera actualizada en este nuevo libro (Nussbaum, 2012: 20-35). 
Tal como Okin subraya, Nussbaum afirma que ambas mujeres están principalmente preocupadas por su propio bienestar físico, económico y social -y el de las personas cercanas a ellas- pero no muestran preocupación por su relación con la naturaleza o por la búsqueda del sentido último de la vida. Lo que Okin está señalando es que no parece haber indicios de que las capacidades listadas por Nussbaum surjan de las necesidades expresadas por las voces de las mujeres que tanto insiste en la necesidad de oír. A pesar de afirmar sin descanso que el enfoque de las capacidades se basa en el respeto por cada individuo entendido siempre como un fin en sí mismo, con valor y dignidad propias, el listado de capacidades y las principales concepciones que lo sostienen parecieran derivar más, a los ojos de Okin, de un ideal aristotélico que de un profundo contacto y conocimiento de las vidas de las mujeres de los países menos desarrollados: «En cuanto a los elementos más sofisticados, incluso extravagantes, en su lista, parecen acercarse más a la vida de una mujer occidental con alto nivel de educación, inclinaciones artísticas, autoconsciente y voluntariamente religiosa, que a las vidas de las mujeres con quienes habló en India» (Okin, 2003: 296). Nussbaum no permitiría a estas mujeres hablar por ellas mismas, pues les estaría imponiendo categorías de su propia inmersión cultural y tradición teórica, dejando de este modo que su voz de intérprete prevalezca sobre la voz de sus entrevistadas. ¿Qué implicaría dentro del enfoque de Nussbaum una preferencia de autoría propia en términos hamptianos?

Nussbaum dirá que existen aproximaciones teóricas que derivan los principios políticos de modo directo de las voces y testimonios de las personas, pero que tienen serios problemas para lidiar con el fenómeno de las preferencias adaptativas. La propia Okin, muy crítica de Nussbaum en este aspecto, afirmaba en un texto suyo anterior que personas sometidas a condiciones injustas adaptan en algunos casos sus preferencias como modo de encubrir ante ellas mismas la injusticia de su situación: «A menudo, las personas oprimidas han interiorizado de tal forma su opresión que no tienen sentido alguno de aquello a que tienen justo derecho como seres humanos» (Okin, 1996: 201).

Debemos señalar también las precauciones que Nussbaum toma en el análisis de la formación de las preferencias de las personas. La autora dedica todo un capítulo de Las mujeres y el desarrollo humano a reflexionar sobre el tema, y parece sostener que no todas las preferencias adaptativas son malas, pero que aquellas que emergen de la injusticia y la subordinación sí lo son. Un enfoque que contemplara estas preferencias sin cuestionarlas estaría manteniendo y reforzando el statu quo. Habría dos maneras, en Nussbaum, de abordar la cuestión: por un lado, las preferencias adaptativas podrían ser muestras de expectativas responsables y realistas por parte de las personas en cuanto a aquello a lo que pueden aspirar, pero por otro, también podrían representar deformaciones en los deseos y elecciones de las personas afectados por hábitos, miedos o condiciones de vida injustas. Pareciera entonces que las preferencias adaptativas solo serían reprobables cuando implican ajustarse a malas condiciones o circunstancias. En cualquier caso, la cuestión crea varios inconvenientes al interior de una teoría como la de Nussbaum, que se erige sobre la libertad individual de elección y en el objetivo de que todas y cada una de las voces cuenten e importen. 
Si bien resulta razonable ser cuidadosa cuando de analizar las elecciones y preferencias de las personas se trata, dado que éstas se encuentran socialmente construidas y condicionadas por múltiples factores; resulta no obstante paradójico, como señala Anne Phillips, incorporar estas consideraciones en el marco de una filosofía que hace de la capacidad de elección la característica definitoria de lo humano: «Nussbaum se encuentra en una postura en la que a la vez está defendiendo a rajatabla la idea de la elección y se muestra crítica de la mayoría de las elecciones que las personas hacen» (Phillips, 2009: 148). Phillips resalta esta tensión para realizar la pregunta que guía de algún modo este trabajo: ¿es posible compatibilizar un análisis sobre la formación social de las preferencias con la primacía liberal de la elección?

A su vez, el énfasis puesto, como ya hemos visto, en una idea intuitiva de lo que es una vida humana digna, no se reconciliaría fácilmente con una mirada crítica sobre las preferencias: ¿cómo separar las ideas intuitivas de los deseos y elecciones de las personas? Lo que nos haría preguntarnos entonces ¿de quiénes son las ideas intuitivas que sostienen al enfoque? ¿Qué ideas intuitivas importan? Resulta pertinente cuestionar si existen acaso preferencias que no estén moldeadas en algún grado por el sistema de género en el que estamos inmerses. ¿Hay preferencias que no son adaptativas? Las diversas personas que han participado del proceso de formulación de la lista de capacidades que presenta Nussbaum, también han sido criadas y socializadas en un contexto de sexismo y opresión, por lo que sería lícito pensar que han internalizado situaciones de subordinación que se expresan en el listado -a través del hecho de privilegiar ciertas capacidades, o de la omisión de otras. Por más que Nussbaum se esfuerce por evitar la acusación de neocolonialismo, partes de su teoría, como esta, le dificultan la tarea.

La oposición de Nussbaum al relativismo aparece en múltiples ocasiones a lo largo de su obra, motivada en la consciencia de que posturas de ese tipo muchas veces racionalizan y justifican injusticias hacia las mujeres en distintos rincones del planeta. Sin embargo, no parecen tan convincentes sus argumentos a la hora de demostrar que las capacidades que conforman su lista gozan de un acuerdo transcultural internacional. Los esfuerzos por evitar el relativismo, no tienen un correlato cuando se trata de sortear los peligros del subjetivismo o incluso de la arbitrariedad. El mayor problema reside en que cuando quien se arroga la autoridad de definir valores universales pertenece a un grupo privilegiado y se encuentra en una posición de poder, los riesgos de caer en un elitismo moral o de ser acusada de neocolonialismo son aún mayores ${ }^{8}$. Observemos, por ejemplo, esta afirmación:

En primer lugar, me parece muy importante que gente de una gran diversidad de culturas, coincidiendo en las condiciones que conducen a una crítica reflexiva de la tradición y libres de toda intimidación o jerarquía, estén de acuerdo en que esta lista es adecuada, siendo la que ellos elegirían. El hecho de encontrar estas áreas de un consenso informado es valioso desde el punto de vista epistémico, y lo es en dos sentidos: en primer término, nos señala áreas de la expresión humana 
que podemos haber descuidado o subestimado. En segundo término, nos indica que nuestras intuiciones acerca de lo que haría posible un consenso político están en la senda correcta (Nussbaum, 2002: 210).

Este pasaje condensa aquello que venimos señalando como problemático en el planteo de Nussbaum: las personas referidas como «gente de una gran diversidad de culturas» aparecen como testigos que, en caso de acordar con un «nosotres» enunciador, valida nuestras afirmaciones, y en caso de diferir, nos permite desestimar sus preferencias en tanto poco fiables o mal informadas. Y como afirma Alison Jaggar, «[c]uando «nosotres» remite a filósofes occidentales y les otres en cuestión son no-filósofes del Tercer Mundo, esto puede quedar incómodamente cerca de tratarles como «informantes natives»» (Jaggar, 2006: 319). Nussbaum no parece percibir su propia posición jerárquica y la intimidación que esta pudiera causar a sus interlocutoras, ni parece someter en ningún momento a consideración que sus propias intuiciones o preferencias pueden estar equivocadas o resultar engañosas.

El objetivo puede ser loable: allí donde las condiciones de injusticia y desigualdad son tales que hacen que las expectativas y ambiciones de las mujeres sean extremadamente bajas, es tarea de una teoría de la justicia feminista elevarlas. Hasta que las mujeres, lesbianas, travestis, trans, bisexuales y no binaries no dispongan del mismo abanico de opciones que los hombres, las mismas oportunidades y expectativas de resultados, parece difícil asegurar hacia dónde podrían dirigirse sus decisiones, cuál sería el proceso de su toma y cómo sería la experiencia en su conjunto. Pero la desconfianza hacia las preferencias que puedan expresar estas sujetas en el marco de un enfoque que pretende encarnar los deseos y aspiraciones de las mujeres a nivel mundial, presenta un grave problema para la coherencia de su propuesta feminista.

Como hemos visto, las preferencias adaptativas parecen representar un dilema para los feminismos liberales -como hemos podido esbozar en los casos de Hampton, Okin y Nussbaum - ya que el componente liberal las guía a valorizar la libertad de elección individual y el componente feminista las hace receptivas a las formas en que la subordinación y la opresión afectan la percepción de las personas acerca de su valor y sus derechos ${ }^{9}$. Esta tensión en torno a las preferencias, ¿hace del feminismo y el liberalismo dos campos irreconciliables? ${ }^{10}$

\section{Preferir y elegir no son lo mismo}

Tal vez Ann Cudd, teórica también posicionada en el feminismo liberal y crítica de algunos aspectos del enfoque nussbaumiano, nos permita comenzar a salir

9 Podríamos, siguiendo a Nussbaum, matizar este comentario. La autora afirma que no sólo la tradición marxista y la noción de «falsa consciencia» han influenciado la crítica feminista del deseo, ya que «la idea de que algunas preferencias están deformadas por la ignorancia, la malicia, la injusticia y el hábito ciego tienen hondas raíces también en la tradición liberal de filosofía política» (Nussbaum, 2002: 165). Como ejemplos nombra a Adam Smith, Mill, Kant, Rawls, y más recientemente Amartya Sen, Jon Elster y Gary Becker.

10 Véase por ejemplo el texto «Liberalism, Adaptive Preferences, and Gender Equality» de Ann Levey (2005). 
de esta encrucijada con su propuesta de distinción entre las preferencias y las elecciones. Desde una perspectiva no-feminista, sostiene Cudd, pueden confundirse los incentivos sociales para tomar ciertas decisiones con efectos en lugar de causas de las elecciones de las mujeres; pero reconocerlas como tal no invalida que tanto las preferencias como las elecciones son elementos centrales del liberalismo. El hecho de que resulten tan problemáticas señala la necesidad de que sean evaluadas tanto por su racionalidad como por su pertinencia en el marco de una sociedad liberal y feminista. En este sentido Cudd sostiene que no todas las preferencias pueden ser respetadas: «Lo que propongo es hacer un mejor trabajo del que los teóricos no-feministas han hecho en la selección de un conjunto de preferencias, o una zona de privacidad, que ha de ser respetada» (Cudd, 2004: 54). El modo en el que la autora puede conciliar la exclusión de ciertas preferencias con la protección de la libertad individual radica en la propia definición de liberalismo; es decir, definir al liberalismo de modo que específicamente excluya las preferencias que causan o reproducen la opresión.

A partir de la diferencia entre fuerzas directas -aquellas que afectan las elecciones de los individuos: la violencia física y psicológica, la discriminación o los incentivos económicos- y fuerzas indirectas - moldean las creencias y deseos, a la vez que nuestras percepciones y comportamiento hacia otres: incluyen los marcadores de género, raza o etnia que elegimos para identificarnos con un grupo social determinado y para excluir, estereotipar y crear prejuicios sobre otros grupos en diversas formas-, Cudd distingue dos tipos de situaciones sociales y políticas. Las personas actúan de acuerdo a sus preferencias en situaciones informales y en situaciones formales. En las situaciones informales las acciones de las personas no están encuadradas en reglas determinadas, por lo que las sanciones para eventuales acciones «indeseables» son difusas. En el caso de las situaciones formales, hay una adhesión a reglas y normas determinadas y explícitas, y las consecuencias de violar esas reglas también están explicitadas y son impuestas institucionalmente.

La propuesta de Cudd es restringir en las situaciones formales las preferencias que refuerzan la opresión del mismo modo que deben restringirse las definidas como iliberales, es decir, aplicando sobre ellas sanciones y desincentivos. Pero en el caso de las situaciones informales, la autora sostiene que debemos oponernos a ellas en modos también informales e indirectos, pero no mediante mecanismos formales: «Contrariamente al espíritu de la lista de capacidades, sostengo que no podemos resolver directamente este problema, porque nuestra propia imaginación moral ha sido atrofiada por la opresión» (Cudd, 2004: 56). De este modo, el aporte a la solución del problema estaría dado por fomentar una «imaginación expansiva» -y aquí sí sirve de ayuda el listado de las capacidades, pues requiere el estímulo y sostenimiento de las condiciones para el uso de la imaginación en modos expansivos. El dominio de la imaginación implica ni más ni menos que nuestra capacidad para imaginar modos diferentes de ser, modos que no se encuentren constreñidos por las normas y estructuras en las que nos encontramos inmersas.

Ahora bien, Cudd se realiza la pregunta del millón: ¿de qué modo abolir la opresión sin violar las preferencias de quienes se encuentran oprimidas? La respuesta que ofrece se fundamenta en los cambios graduales: «necesitamos dar pa- 
sos pequeños y transformadores hacia la libertad en lugar de realizar un esfuerzo directo por reformar las preferencias» (Cudd, 2004: 58).

La respuesta de Nussbaum sería claramente diferente. Las preferencias de las personas pueden guiarnos hacia ciertos temas o problemas de interés, pero le debemos dar a los deseos un rol modesto en las justificaciones políticas, "consultando al deseo informado como un control cruzado en nuestro argumento moral independiente» (Nussbaum, 2004: 200). Sin negar el papel que los deseos pueden jugar en los principios políticos, lo que se debería dejar en claro es que el argumento moral independiente debe primar. Otra forma de lidiar con esta pregunta es la que propone Okin bajo la figura que denomina «commited outsiders».

Después de todo, la distancia crítica no tiene por qué conllevar el desinterés: personas foráneas pero comprometidas a menudo pueden ser mejores analistas y críticos de la injusticia social que las personas que viven en la cultura donde estas injusticias se producen. De ahí que un concepto como la posición original, que pretende aproximarse a un punto arquimedeano, sea tan valioso, al menos como complemento a alguna forma de diálogo (Okin, 1996: 202).

La importancia que, tal como veníamos señalando, parece dar Okin a las voces de las mujeres para dar cuenta de las situaciones de injusticias en las que se ven involucradas, no armoniza muy bien con la noción de preferencias adaptativas. Si muchas mujeres internalizan su situación hasta no percibirla como injusta ¿cuál es el valor de su relato ${ }^{11}$ En este sentido, pareciera más acorde a la idea de preferencias adaptativas esta figura de un/a agente externo/a comprometido/a con la cultura local y con un profundo conocimiento sobre ella, pero con la distancia crítica necesaria para observar las prácticas e instituciones opresivas ${ }^{12}$.

En todo caso, ni la exigencia moral de Hampton, ni el enfoque político de Nussbaum, ni las figuras intermediarias de Okin, ni los cambios graduales de Cudd terminan de despejar los problemas o de dar alguna respuesta un poco más clara a las tensiones que observamos entre la primacía liberal de la libre elección y la formación social, económica, cultural, política, de las preferencias.

\section{Para concluir, tensiones}

Tal vez, llegadas aquí, nos encontremos ante la situación en la que incluso la mejor versión del feminismo liberal, no nos resulta suficiente para abordar en toda su complejidad el asunto que nos ocupa. Pues si bien representa a una corriente del feminismo que por sus propias concepciones se ha ocupado con denodado interés del desarrollo teórico de los debates en torno a la noción de preferencias adapta-

11 En sus últimos textos, Okin pareciera comenzar a alejarse de la idea de preferencias adaptativas: «una tendría sin duda más posibilidades de conocer y entender las condiciones reales de lo que parece ser la opresión, consultando a las personas que parecen estar oprimidas» (Okin, 2005: 67-89).

12 Este modelo de crítica social híbrida entre un adentro y un afuera, está representado, por ejemplo, en la propuesta de Brooke Ackerly, pensadora feminista enfocada en el estudio de los derechos humanos internacionales y su relación con la teoría democrática y el feminismo (Ackerly, 2000, 2008). 
tivas, y ha revitalizado las conceptualizaciones alrededor de la idea de elección y autonomía, pareciera resultarle imposible ir más allá de las disonancias que se generan a su interior entre sus marcos de referencias.

Pero sostendré que la salida a estas encrucijadas, debemos buscarla justamente en el reconocimiento de las tensiones. Paradojas, aporías y tensiones propias y constitutivas de los feminismos, y que se nos presentan como espacios productivos desde los cuales pensar y actuar.

La teórica Catharine MacKinnon, por ejemplo, no desconoce la complejidad del concepto de libre elección, pero en su reflexión ni las mujeres que experimentan coerción explícita ni aquellas que la padecen en modos más indirectos estarían obrando bajo los influjos de una falsa conciencia (MacKinnon, 1995, 2001). En todo caso se trata de respuestas reales a contextos reales, y todas deben ser tomadas en cuenta. Pero no puede perderse de vista que esas respuestas se dan en el marco y como resultado de condiciones opresivas ${ }^{13}$. A pesar de que resulta imposible saber cuáles serían las elecciones de las mujeres en una situación no opresiva, en la teoría de MacKinnon conviven las realidades que coexisten asimismo en cada una de las mujeres: la desigualdad y la autonomía. Las mujeres somos lo que somos y lo que podemos ser más allá de esa realidad de desigualdad. En palabras de Denise Schaeffer: «Si la desigualdad sexual no fuera real, el feminismo no existiría. Si fuera una realidad taxativa, el feminismo no podría existir. El feminismo existe en la intersección de la realidad de la opresión de las mujeres y su realidad como seres humanos completos, y afirma la realidad de ambas» (Schaeffer, 2001: 703).

La importancia que la intersección entre la desigualdad y la autodeterminación reviste en el pensamiento de MacKinnon, me parece crucial para alejarnos de ciertas interpretaciones que analizan su obra como una mera victimización constante de las mujeres. Para MacKinnon las mujeres estamos insertas en un sistema social de desigualdad de género, pero no somos enteramente determinadas por él. Es en la dinámica entre estas dos circunstancias donde debemos posicionarnos para analizar la situación. Lejos de celebrar o regodearse en la victimización, la autora resalta las capacidades de elección y autodeterminación que las mujeres poseemos en tanto seres humanos, y reclama una conciencia feminista que descansa en el potencial emancipatorio femenino.

13 La filósofa italiana Michela Marzano cuestiona el argumento liberal que liga de manera directa el consentimiento con la autonomía. Al respecto, la autora afirma: «Aceptemos que se argumente que el consentimiento expresa la autonomía personal y que esta autonomía es un signo de humanidad, hasta el punto que no tomarla en cuenta significaría tratar a un individuo como a un niño, o peor todavía, como un objeto, pero entonces el consentimiento no puede reducirse a la simple manifestación de una necesidad súbita, o de una pulsión incontrolable, o incluso de una necesidad momentánea como si expresa el miedo ante una amenaza física o psíquica -y por ello no puede remitirnos al concepto de autonomía- en este caso el consentimiento no puede justificar una acción o una conducta. Tanto en este caso como en el otro, caemos en «contradicciones lógicas». En el primer caso, en efecto, no se puede exigir a la vez expresar su autonomía y actuar de manera que la autonomía sea puesta en peligro. En el segundo caso, no se puede querer que el consentimiento sea solamente la expresión de una necesidad súbita y a la vez sea considerado como un principio capaz de justificar un acto desde un punto de vista ético» (Marzano, 2009: 37). Marzano desafía de este modo la asunción de que el consentimiento es siempre expresión de autonomía a la vez que la idea de que el consentimiento es una expresión suficiente para legitimar una acción. 
La libertad es socialmente construida, no solo como concepto, sino como práctica, como valor moral, como principio legal. La forma en la que pensamos la libertad, es también un modo situado social e históricamente. Este modo ha sido principalmente masculino, restringiendo no solo las opciones de aquello que las mujeres tenemos "permitido" hacer, sino aquello que se nos deja ser: cómo nos concebimos, qué deseamos, a qué aspiramos, qué preferimos. En palabras de Nancy Hirschmann: «Estas reglas y normas del patriarcado no son simplemente restricciones externas sobre aquello que, de lo contrario, serían deseos naturales de las mujeres; más bien crean todo un contexto cultural que hace que parezca que las mujeres elijen lo que son, en lugar de estar restringidas a ello» (Hirschmann, 2003: 11).

Sin negar la posibilidad de que las personas puedan adaptar sus preferencias a las condiciones de opresión en las que viven, Hirschmann propone la idea de «construcción social» como una mejor forma de acercarse a esta "producción social»: "Al sugerir que las personas se producen a través de las formaciones sociales, y no son simplemente limitadas por ellas, la idea de construcción social pone en tela de juicio la suposición sobre aquello que es genuino o fiel a sí mismo y lo que es falso» (Hirschmann, 2003: 11). Ahora bien, si todes somos y todo es socialmente construido, no solo nuestras restricciones responden a este proceso, sino también nuestras prácticas y acciones. Si bien un análisis de este tipo puede desembocar en un mero relativismo o deconstruccionismo -que termina acercándose demasiado al liberalismo- lo interesante del planteo de Hirschmann es la visibilización de obstáculos tanto externos como internos a la libertad, y el reconocimiento del contexto -social, cultural, institucional, histórico- en el que esas barreras surgen y operan. Para las mujeres, no se trata solo de las amenazas intencionales e identificables a la libertad de cada una de nosotras, del acto sexista perpetrado por un individuo en particular, sino de una cultura patriarcal que asigna mayor valor a los hombres que a las mujeres, más oportunidades y más opciones. El ejemplo de Hirschmann en este sentido resulta iluminador:

Consideremos la decisión de una mujer de salir por la noche. La mayoría de los hombres tienen el poder de violar al menos a algunas mujeres, un «poder en contra de» las mujeres que las mujeres no tienen recíprocamente contra los hombres. La mayoría de los hombres no ejercen este poder, y muchos estarían horrorizados ante la sugerencia de hacerlo, pero es un poder que, sin embargo, impregna la cultura, haciendo que muchas mujeres teman salir por la noche solas. Incluso si ellas mismas nunca han sido violadas o atacadas, el miedo todavía las inhibe. Estas mujeres están privadas de libertad por tal poder y por el miedo que genera; la existencia de un poder "contra», aunque no ejercido en realidad en un momento dado, se ha traducido en un poder generalizado y constante «sobre» (Hirschmann, 2003: 24)

No debemos perder de vista, sin embargo, que somos tanto «sujetas de» como estamos «sujetas a» esas construcciones sociales. Las mujeres no somos receptoras pasivas de reglas externas, sino activas negociadoras de significados en permanente interacción con nuestro contexto. Debemos promover y desarrollar enfoques que 
nos permitan entender la libertad en un contexto de seres situados, y nos demanden prestar atención tanto a la voluntad y deseos internos de cada persona como a los condicionantes sociales externos, o sea, a las relaciones, normas y discursos en los que nos encontramos insertas y sobre los que actuamos. Es decir, es necesario reconocer los modos en que las preferencias de las mujeres han sido históricamente moldeadas por la supremacía masculina, «socialmente construidas» por las instituciones dominantes. Pero a la vez, es imperioso dar cuenta de las prácticas de resistencia ante esas constituciones y construcciones (Hirschmann, 2007), y el modo en que los deseos y voluntades afloran y se expresan aun en condiciones sumamente hostiles.

\section{Referencias bibliográficas}

ACKERLY, Brooke (2008). Universal Human Rights in a World of Difference, Cambridge: Cambridge University Press.

ACKerly, Brooke (2000). Political Theory and Feminist Social Criticism, Cambridge: Cambridge University Press.

Alexander, M. Jacqui y Chandra Talpade Mohanty (2004). «Genealogías, legados, movimientos» en HOOKs bell et al. (2004). Otras inapropiables: Feminismos desde las fronteras, Madrid: Traficantes de sueños, pp. 137-184.

Cudd, Ann (2004). «The Paradox of Liberal Feminism: Preference, Rationality, and Oppression» en BAEHR, Amy (ed.) (2004). Varieties of Feminism Liberalism, Maryland: Rowman \& Littlefield Publishers, pp. 37-61.

Di Tullio, Anabella (2007). «¿Hacia una justicia sin fronteras? El enfoque de las capacidades de Martha Nussbaum y los límites de la justicia» en Daímon. Revista Internacional de Filosofía, No. 58, pp. 51-68.

Hampton, Jean (1993) [2007]. «Selflessness and Loss of Self» en The Intrinsic Worth of Persons. Contractarianism in Moral and Political Philosophy, Cambridge: Cambridge University Press, pp. 39-71.

HirschmanN, Nancy (2007). «Feminist Political Philosophy», en Alcoff, Linda y Kittay, Eva (eds.) (2007). The Blackwell Guide to Feminist Philosophy, Malden: Blackwell Publishing, pp. 145-164.

Hirschmann, Nancy (2003). The Subject of Liberty: Toward a Feminist Theory of Freedom, Nueva Jersey: Princeton University Press.

JAgGAR, Alison (2006). «Reasoning About Well-Being: Nussbaum's Methods of Justifying the Capabilities» en The Journal of Political Philosophy, Vol. 14, No. 3, pp. 301-322.

Levey, Ann (2005). «Liberalism, Adaptive Preferences, and Gender Equality» en Hypatia, Vol. 20, No. 4, pp. 127-143.

MacKinnon, Catharine (2001). ««The Case» Responds» en The American Political Science Review, Vol. 95, No. 3, pp. 709-711.

MacKinnon, Catharine (1995). Hacia una teoría feminista del Estado, Madrid: Ediciones Cátedra. 
Marzano, Michela (2009). Consiento, luego existo. Ética de la autonomía, Capellades: Proteus.

Nussbaum, Martha (2012). Crear capacidades. Propuesta para el desarrollo humano, Barcelona: Paidós.

Nussbaum, Martha (2009). «Las capacidades de las mujeres y la justicia social» en Debate Feminista, Año 20, Vol. 39, pp. 89-129.

Nussbaum, Martha (2007). Las fronteras de la justicia: consideraciones sobre la exclusión, Barcelona: Paidós.

Nussbaum, Martha (2004). «On Hearing Women's voices: A Reply to Susan Okin» en Philosophy \& Public Affairs, Vol. 32, No. 2, pp. 193-205.

Nussbaum, Martha (2000). Las mujeres y el desarrollo humano: el enfoque de las capacidades, Barcelona: Herder.

OкIN, Susan Moller (2005). «Multiculturalism and Feminism: No Simple Questions, No Simple Answers», en EISEnBerg, Avigail y SpInNer-Halev, Jeff (eds.) (2005). Minorities within Minorities: Equality, Rights and Diversity, Cambridge: Cambridge University Press, pp. 67-89.

ОкіN, Susan Moller (2003). «Poverty, Well-Being, and Gender: What Counts, Who's Heard?» en Philosophy \& Public Affairs, Vol. 31, No. 3, pp. 280-316.

ОкіN, Susan Moller (1996). «Desigualdad de género y diferencias culturales» en Castells, Carme (comp.) (1996). Perspectivas feministas en teoría política, Barcelona: Paidós, pp. 185-206.

Phillips, Anne (2009). «El feminismo y el liberalismo nuevamente: ¿tiene razón Martha Nussbaum?» en Debate Feminista, Vol. 39, pp. 130-151.

Rawls, John (1995). El liberalismo político, México D.F.: Fondo de Cultura Económica.

SCHAEFfER, Denise (2001). «Feminism and Liberalism Reconsidered: The Case of Catharine MacKinnon» en The American Political Science Review, Vol. 95, No. 3, pp. 699-708.

Sen, Amartya (1998). «Capacidad y bienestar» en Nussbaum, Martha y Sen, Amartya (comps.) (1998). La calidad de vida, México D.F.: Fondo de Cultura Económica, pp. 54-83.

SEn, Amartya (2011). La idea de la justicia, Buenos Aires: Taurus.

Recibido el 9 de mayo de 2020 Aceptado el 3 de diciembre de 2020 BIBLID [1132-8231 (2021): 251-265] 